\title{
Evaluation of Flavonoids Binding to DNA Duplexes by Electrospray Ionization Mass Spectrometry
}

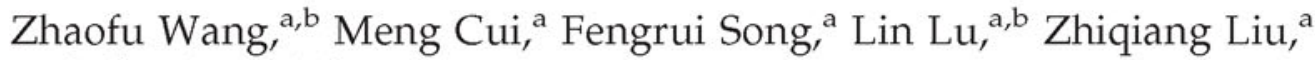 \\ and Shuying Liu ${ }^{a}$ \\ ${ }^{a}$ Changchun Institute of Applied Chemistry, Chinese Academy of Sciences, Changchun Center of Mass \\ Spectrometry, Changchun, 130022, P. R. China \\ ${ }^{\mathrm{b}}$ Graduate School of the Chinese Academy of Sciences, Beijing, 100039, P. R. China
}

In this study, electrospray ionization mass spectrometry (ESI-MS) was used to investigate the binding interactions of ten flavonoid aglycones and ten flavonoid glycosides with DNA duplexes. Relative binding affinities of the flavonoids toward DNA duplexes were estimated based on the fraction of bound DNA. The results revealed that the 4'-OH group of flavonoid aglycones was essential for their DNA-binding properties. Flavonoid glycosides with sugar chain linked on ring A or ring B showed enhanced binding toward the duplexes over their aglycone counterparts, whereas glycosylation of the flavonol quercetin on ring $\mathrm{C}$ exhibited a less pronounced effect. The aglycone skeletons and other hydroxyl substitutions on the aglycone also have an effect on the fractions of bound DNA. Upon collision-induced dissociation, the complexes containing flavonoid aglycones underwent the predominant ejection of a neutral ligand molecule, suggesting an intercalative DNA-binding mode. However, for complexes containing flavonoid glycosides, the loss of nucleobase increased to different extents, indicating a stronger binding or different binding mode. The results may provide not only a deeper insight into the DNA-binding properties of flavonoids but also a useful guideline for the design of efficient DNA-binding agents for chemotherapy. (J Am Soc Mass Spectrom 2008, 19, 914-922) (C) 2008 American Society for Mass Spectrometry

$\bigcirc$ mall molecules binding to nucleic acid structures are of great interest in modern medicine because they constitute a significant portion of the anticancer drugs [1]. These drugs may bind to certain DNA sequences, prevent the specific recognition of these sequences with their trans-acting factors, and interfere with the replication or transcription of certain genes, and thus exert their pharmacological activities [2]. Among the various organic molecules, natural products have attracted considerable interest given that many clinical anticancer drugs are natural products (or their derivatives) and most of them exert their effects by acting on DNA [1]. In this context, studies of the binding of natural products with DNA are helpful for better understanding the molecular basis of their bioactivities as well as providing useful guidance for further design of more efficient anticancer drugs [1, 3-7].

As an important class of natural products, flavonoids have received considerable attention because of their health benefits and chemopreventive properties [8]. Flavonoids consist of a diverse group of compounds derived from 2-phenolchromotome and can be categorized into several subgroups according to the structure

Address reprint requests to Dr. Zhiqiang Liu, Changchun Institute of Applied Chemistry, Chinese Academy of Sciences, Changchun Center of Mass Spectrometry, Changchun, Jilin 130022, China. E-mail: liuzq@ciac.jl.cn of the C-ring, such as flavone, flavonol, flavanone, isoflavone, and flavan-3-ol (Table 1). Flavonoid aglycones and their modified forms are widely found in the roots, stalks, and fruits of many natural plants and act as the major functional components in many herb medicines [8,9]. Many studies have demonstrated that flavonoids possess a wide range of biological activities, such as anticancer, antiviral, antibacterial, antioxidants, and anti-inflammatory effects [8]. These biological activities are considered to be related to their interaction with several enzymes in the human body as well as their notable antioxidant activity [8, 10]. Meanwhile, the binding of flavonoids to nucleic acid structures has also been recognized as one important mechanism of their actions [11-19].

The noncovalent interactions between flavonoids and DNA have been elucidated using several solution-phase techniques including absorption [20, 21], fluorescence [21], linear dichroism [22], and nuclear magnetic resonance [23] spectroscopies, as well as electrochemical [24] and surface plasmon resonance [25] techniques. However, these methods are laborintensive or require large quantities of materials. Electrospray ionization mass spectrometry (ESI-MS) has proven to be a powerful tool for examining the drug/DNA complexes because the intact complexes can survive the gentle ionization process and be 
Table 1. Structures of the flavonoids investigated in this study

\begin{tabular}{|c|c|c|c|c|}
\hline Structure & Name & Nomenclature & Mass & Note $^{a}$ \\
\hline & $\begin{array}{l}\text { Baicalein } \\
\text { Apigenin } \\
\text { Luteolin } \\
\text { Baicalin } \\
\text { Vitexin }\end{array}$ & $\begin{array}{l}\text { 5,6,7-Trihydroxyflavone } \\
5,7,4^{\prime} \text {-Trihydroxyflavone } \\
5,7,3^{\prime}, 4^{\prime} \text {-Tetrahydroxyflavone } \\
\text { Baicalein-7-O-glucuronic acid } \\
\text { Apigenin-8-C-glucoside }\end{array}$ & $\begin{array}{l}270 \\
270 \\
286 \\
446 \\
432\end{array}$ & $\begin{array}{l}\text { FA } \\
\text { FA } \\
\text { FA } \\
\text { FG } \\
\text { FG }\end{array}$ \\
\hline & $\begin{array}{l}\text { Quercetin } \\
\text { Quercetrin } \\
\text { Hyperin } \\
\text { Rutin }\end{array}$ & $\begin{array}{l}3,5,7,3^{\prime}, 4^{\prime} \text {-Pentahydroxyflavone } \\
\text { Quercetin-3-O-rhamnoside } \\
\text { Quercetin-3-O-galactoside } \\
\text { Quercetin-3-O-rhamnosylglucoside }\end{array}$ & $\begin{array}{l}302 \\
448 \\
464 \\
610\end{array}$ & $\begin{array}{l}\text { FA } \\
\text { FG } \\
\text { FG } \\
\text { FG }\end{array}$ \\
\hline & $\begin{array}{l}\text { Liquiritigenin } \\
\text { Naringenin } \\
\text { Liquiritin } \\
\text { Naringin }\end{array}$ & $\begin{array}{l}\text { 7,4'-Dihydroxyflavanone } \\
\text { 5,7,4'-Trihydroxyflavanone } \\
\text { Liquiritigenin-4'-O-glucoside } \\
\text { Naringenin-7-O-rhamnosylglucoside }\end{array}$ & $\begin{array}{l}256 \\
272 \\
418 \\
580\end{array}$ & $\begin{array}{l}\text { FA } \\
\text { FA } \\
\text { FG } \\
\text { FG }\end{array}$ \\
\hline ne & $\begin{array}{l}\text { Daidzein } \\
\text { Formononetin } \\
\text { Genistein } \\
\text { Puerarin } \\
\text { Daidzin } \\
\text { Genistin }\end{array}$ & $\begin{array}{l}\text { 7,4'-Dihydroxyisoflavone } \\
\text { 7-Hydroxy-4'-methoxyisoflavone } \\
\text { 5,7,4'-Trihydroxyisoflavone } \\
\text { Daidzein-8-C-glucoside } \\
\text { Daidzein-7-O-glucoside } \\
\text { Genistein-7-O-glucoside }\end{array}$ & $\begin{array}{l}254 \\
268 \\
270 \\
416 \\
416 \\
432\end{array}$ & $\begin{array}{l}\text { FA } \\
\text { FA } \\
\text { FA } \\
\text { FG } \\
\text { FG } \\
\text { FG }\end{array}$ \\
\hline 3-ol & (-)-Catechin & & 290 & FA \\
\hline
\end{tabular}

a"FA" and "FG" denote, respectively, flavonoid aglycones and flavonoid glycosides.

maintained in the gas phase [26]. Advantages such as low sample consumption and fast analysis time also render ESI-MS suitable for high-throughput screening [27]. To date, many well-characterized drug/ DNA complexes have been extensively studied by ESI-MS [28-44]. However, the application of ESI-MS to characterize the flavonoid/DNA complexes has been involved only in a recent work for detection of the daidzin/G-quadruplex complex [45].
The purpose of the present study is to evaluate the binding of flavonoids to DNA duplexes for the first time using ESI-MS. Five DNA sequences were involved (Table 2): two critical Sp-1 binding sites in human surviving promoter (duplexes 1 and 2) were selected as in our previous study [5]; the other three sequences with different GC and AT content (duplexes 3, 4, and 5) were also selected to further investigate possible sequence selectivities of the flavonoids. Binding sto-

Table 2. Oligodeoxynucleotides used in this study

\begin{tabular}{llr}
\hline Name & Sequence & Mass (Da) \\
\hline \hline Duplex 1 & d(AACTCCCGGCACAC/GTGTGCCGGGAGTT) (ss1a/ss1b) & 8524.5 \\
Duplex 2 & d(ACGCGGCGGGAGGA/TCCTCCCGCCGCGT) (ss2a/ss2b) & 8526.5 \\
Duplex 3 & d(GGCGAAATTGCGG/CCGCTTTAAACGCC) (ss3a/ss3b) & 8523.5 \\
Duplex 4 & d(GGCGGAATTCGCGG/CCGCCTTAAGCGCC) (ss4a/ss4b) & 8525.5 \\
Duplex 5 & d(GGCGGGATCCGCGG/CCGCCCTAGGCGCC) (ss5a/ss5b) & 8527.5 \\
\hline
\end{tabular}


ichiometries were easily obtained from the negativeion mass spectra. Relative binding affinities of the flavonoids for the DNA duplexes were also evaluated and structural features for their DNA-binding properties were elucidated. Finally, the stabilities of the flavonoid/DNA complexes were examined using collision-induced dissociation (CID) experiments. The results demonstrate that ESI-MS is a powerful tool for investigation of the interactions between flavonoids and DNA.

\section{Experimental}

\section{Materials}

Milli-Q ${ }^{\mathrm{TM}}$ water (Millipore, Bradford, MA, USA) was used in all the experiments. Methanol was of HPLC grade from Fisher Chemicals (Fair Lawn, NJ, USA). All the flavonoids (Table 1) were obtained from the National Institute for the Control of Pharmaceutical and Biological Products (Beijing, China). Stock solutions of the flavonoids were prepared in methanol at a concentration of $1 \mathrm{mM}$.

Single-strand oligodeoxynucleotides (ODNs) were purchased from Takara Biotechnology Co., Ltd. (Dalian, China) and used without further purification. Stock solutions of each ODN were prepared at $2 \mathrm{mM}$ concentration in Milli-Q water. Annealing was achieved by preparing solutions containing two complementary ODN strands in $500 \mathrm{mM}$ ammonium acetate. For duplexes 1, 3, 4, and 5, equal volumes of each complementary ODN were mixed. However, for duplex 2, the volume ratio of ss2a to ss $2 b$ was adjusted to $2: 1$ to ensure that the duplex ion was predominant in the spectrum. The annealing solutions were heated to $90^{\circ} \mathrm{C}$ for $15 \mathrm{~min}$ and slowly cooled at $4{ }^{\circ} \mathrm{C}$ overnight to form the DNA duplexes. Concentrations of the single strands were estimated by UV spectroscopic measurements at $260 \mathrm{~nm}$ and the extinction coefficients provided by the manufacturer.

For analysis, solutions containing one duplex and one flavonoid with a $1: 4 \mathrm{M}$ ratio $(20$ and $80 \mu \mathrm{M}$, respectively, except for the concentration-dependent study) were prepared in methanol/50 $\mathrm{mM}$ ammonium acetate $(1: 3, \mathrm{vol} / \mathrm{vol})$ and allowed to equilibrate at room temperature.

\section{Mass Spectrometry}

All the mass spectra were collected on a Finnigan LCQ ion trap mass spectrometer (ThermoFinnigan, San Jose, CA, USA) in negative-ion mode. The sample solutions were infused into the mass spectrometer via a Harvard syringe pump (Holliston, MA, USA) at a flow rate of 3 $\mu \mathrm{L} / \mathrm{min}$. The spray voltage was set to $-3.0 \mathrm{kV}$. The heated capillary temperature was set at $140{ }^{\circ} \mathrm{C}$ and nitrogen sheath and auxiliary gas flows of 50 and 15 arbitrary units, respectively, were used to ensure efficient desolvation. Other instrumental conditions were autotuned using the free duplex ions. Spectra were acquired by summing 30 scans.

CID experiments were performed on selected complexes by isolating the desired precursor ion in the ion trap, followed by fragmentation promoted with increment of the collision energy until the intensity of the precursor ion was reduced to approximately $10-20 \%$ of its original abundance. A default activation time of 30 ms was used during all the measurements.

\section{Results and Discussion}

\section{Binding of Flavonoid Aglycones to DNA Duplexes}

The interactions between flavonoid aglycones and DNA duplexes have been extensively studied by several solution-phase methods [20-25]. In this study, complexation of the aglycones with the duplexes was first evaluated by negative-ion ESI-MS to demonstrate that results obtained by MS can be correlated to the solution-binding behaviors.

Solutions containing one flavonoid aglycone and one duplex at a 4:1 $\mathrm{M}$ ratio (80 and $20 \mu \mathrm{M}$, respectively) were analyzed using negative-ion ESI-MS, and representative mass spectra are shown in Figure 1a-e. Among these aglycones, quercetin and genistein have long been recognized as efficient topoisomerase poisons and their binding to DNA structures has been extensively studied [20-24]. In the negative-ion mass spectra, peaks corresponding to the 1:1 and 2:1 flavonoid/DNA complexes were clearly observed for these two aglycones (Figure 1c and e). The same binding stoichiometries were obtained for the other flavonoid aglycones (except for baicalein and formononetin), with complex ions above $5 \%$ relative intensities.

In contrast, for solutions containing the aglycone baicalein or formononetin, peaks corresponding to the flavonoid/DNA complexes were not detected (Figure 1a), indicating rather weak interactions between the duplex and these two flavonoid aglycones. This observation was in agreement with previous results obtained using fluorescence intercalator displacement (FID) assay [18]. The absence of DNA complex ions with baicalein or formononetin could also be used to preclude the formation of nonspecific aggregates between the flavonoids and DNA duplexes in the gas phase under the present experimental conditions.

The binding results of the aglycones with the other four duplexes were similar to those obtained for the duplex 1, suggesting that these aglycones do not have significant sequence selectivities. Therefore, these results have demonstrated that ESI-MS is indeed an effective method for screening the DNA-binding properties of the flavonoid ligands.

\section{Binding of Flavonoid Glycosides to DNA Duplexes}

In plants, various modified forms of flavonoid aglycones also exist and exert a wide range of biological 

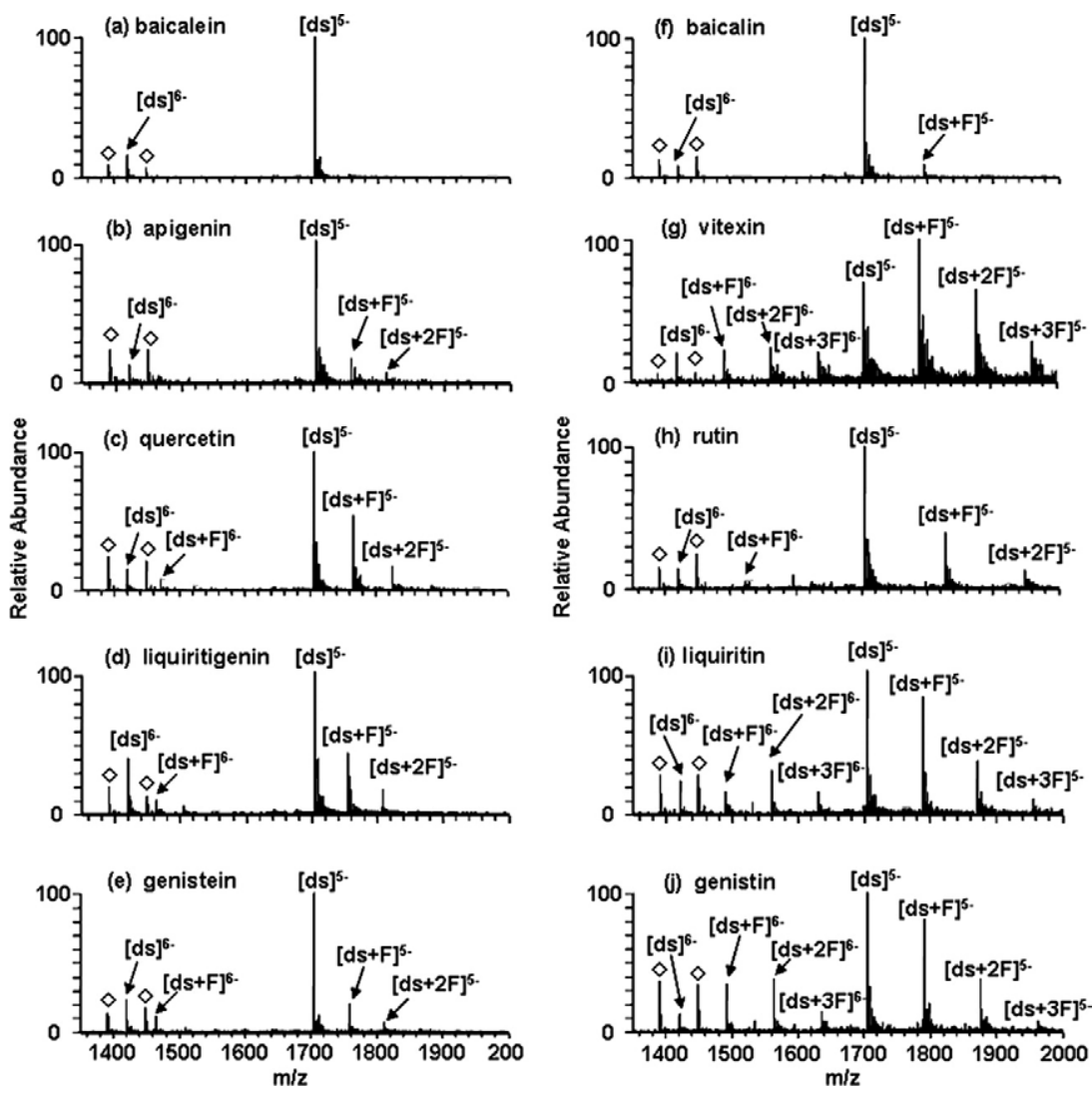

Figure 1. Representative ESI mass spectra of solutions containing a 1:4 ratio of duplex 1 with flavonoid aglycones $(\mathbf{a}-\mathbf{e})$ and flavonoid glycosides $(\mathbf{f}-\mathbf{j})$. Ions from single-stranded DNA are marked with $\diamond$.

activities. Among them, flavonoid glycosides are recognized as the most important modified form. Flavonoid O-glycosides exist commonly with one or more hydroxyl groups of the aglycone linked with a sugar chain to form a glycosidic $\mathrm{O}-\mathrm{C}$ bond. In addition, glycosylation can also occur by direct linkage of the sugar chain to the flavonoid's basic nucleus via a $\mathrm{C}-\mathrm{C}$ bond, forming flavonoid C-glycosides. The flavonoid glycosides exhibit various biological activities including anticancer, anti-HIV, and antiviral effects [8, 9]. In principle, any of the hydroxyl groups and basic nucleus in the aglycones can be glycosylated; however, certain positions are favored: for example, the 7- and/or $4^{\prime}-\mathrm{OH}$ group in flavones, flavanones, and isoflavones; the $3-\mathrm{OH}$ group in flavonols; and the C-6 and/or C-8 position of the flavonoid nucleus [9]. Compared with the flavonoid aglycones, binding interactions of flavonoid glycosides with nucleic acid structures have been investigated less frequently.

Solutions containing a $4: 1$ ratio ( 80 and $20 \mu \mathrm{M}$, respectively) of one flavonoid glycoside and one duplex were also examined by negative-ion ESI-MS. All the flavonoid glycosides studied were found to form complexes with the DNA duplexes, exhibiting a variety of binding stoichiometries as demonstrated in Figure 1f-j. For the flavone glycuronide baicalin, only the complex with 1:1 binding stoichiometry was observed (Figure 1f). In the case of the flavonoid diglycosides rutin and naringin, the 1:1 and 2:1 flavonoid/DNA complexes could be observed (Figure 1h). For the remaining flavonoid glycosides, the flavonoid/DNA complexes showed binding stoichiometries ranging from 1:1 and $3: 1$. It is worth noting that, in the mass spectrum obtained for the flavone glycoside vitexin (Figure 1g), the 1:1 flavonoid/DNA complex was the most abundant, whereas the abundance of 2:1 complex was nearly equal to that of free duplex. However, for the other flavonoid glycosides, the abundances of the complexes were lower than that of the free duplex. These results suggested that vitexin undergoes more extensive complexation with the DNA duplex than the other flavonoid glycosides, which may be attributed to the specific structure of vitexin (see discussion in the next section). Similar binding results were obtained for the complexes of the other four DNA duplexes with these flavonoid glycosides.

\section{Relative Binding Affinities of the Flavonoids to DNA Duplexes}

The relative binding affinities of the flavonoids toward the DNA duplexes were compared using the values of 
fraction of bound DNA, which were calculated using the following equation:

$$
\begin{aligned}
& \text { Fraction of bound DNA }=\left(\mathrm{I}_{(1: 1)}+\mathrm{I}_{(1: 2)}+\mathrm{I}_{(1: 3)}\right. \\
& +\cdots) /\left(\mathrm{I}_{\mathrm{DNA}}+\mathrm{I}_{(1: 1)}+\mathrm{I}_{(1: 2)}+\mathrm{I}_{(1: 3)}+\cdots\right)
\end{aligned}
$$

where $\mathrm{I}_{\mathrm{DNA}}$ is the relative abundance of the free DNA and $\mathrm{I}_{(1: n)}$ represents the relative abundances of the DNA/flavonoid complexes in the ESI mass spectra. With an assumption that the ESI response factors of the free DNA and the drug/DNA complexes are identical, the relative intensities of the free and bound DNA in the mass spectra are expected to be proportional to their relative abundances in solution [34]. Therefore, the values of fraction of bound DNA can be used as a unique parameter to compare the relative binding affinities of drugs with DNA structures [41-43]. Higher values of fraction of bound DNA indicate greater DNAbinding affinities. It has also been demonstrated in several studies that the relative binding affinities measured by ESI-MS were in fairly good agreement with the results obtained using solution-phase techniques [41-43]. Here, the relative binding affinities of the flavonoids toward duplex 1 are shown in Figure 2 as an example. The results for the other four duplexes followed the same trend (data not shown).

It can be seen from Figure 2 that the $4^{\prime}-\mathrm{OH}$ group of flavonoid aglycones is essential for their DNA-binding properties. For example, both baicalein and apigenin contain three $\mathrm{OH}$ groups, two of which are at the 5 and 7 positions of ring A. The only difference between these two molecules is the position of the third $\mathrm{OH}$ group, that is, baicalein has a $6-\mathrm{OH}$, whereas apigenin has a $4^{\prime}-\mathrm{OH}$; this subtle difference resulted in a significant

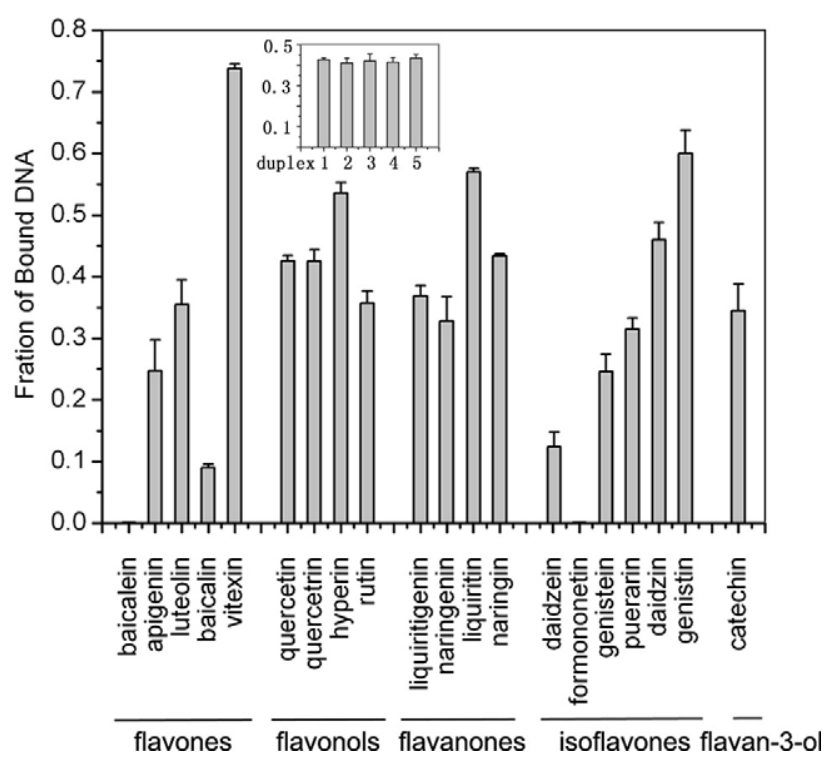

Figure 2. Relative binding affinities of the flavonoids to duplex 1 based on ESI-MS measurements. Inset shows the relative binding affinities of quercetin to the five duplexes. The molar ratio of flavonoid to DNA was 4:1. increase in the fraction of bound DNA of apigenin. Replacement of the $\mathrm{OH}$ group by the $\mathrm{OCH}_{3}$ group at the 4 '-position in the pair daidzein/formononetin, however, led to a notable decrease in the fraction of bound DNA. These results suggested that the $4^{\prime}-\mathrm{OH}$ is of primary importance for significant DNA binding of the flavonoid aglycones, just as revealed in previous studies $[12,17,19]$. This hydroxyl group may form a hydrogen bond with the DNA structures and stabilize the complexes [17].

Glycosylation on the flavonoid aglycones was proved to be related to the flavonoids binding to the DNA duplexes. For the flavonoids used in this study, glycosylation on either ring A or ring B of the aglycones, regardless of the identity of the sugar chain, could undoubtedly result in a significant increase of the DNA-binding affinities. As seen in Figure 2, the flavonoid glycosides with sugar chains conjugated on either of these two rings show much higher fractionbound values than their aglycone counterparts. It is not surprising that glycosylation on the $4^{\prime}-\mathrm{OH}$ group in liquiritin can lead to increased DNA-binding affinity over the aglycone liquiritigenin because the conjugated glucose can provide the necessary stabilization instead of the $4^{\prime}-\mathrm{OH}$ in liquiritigenin and further enhance the interactions by forming extra hydrogen bonds with the DNA duplexes. Compared with rings $\mathrm{A}$ and $\mathrm{B}$, glycosylation on ring $\mathrm{C}$ (the $3-\mathrm{OH}$ group of flavonol) seems to have a less pronounced effect on the DNA-binding affinities. In our study, the flavonol glycoside quercetrin possessed approximately the same binding affinity as that of its aglycone quercetin; hyperin showed a slightly increased affinity toward the duplex, whereas rutin exhibited a slight decrease of binding affinity. Because these three flavonoid glycosides have the same flavonol aglycone and differ only in the glycoside moieties, the fraction-bound value differences suggest that the sugar chain can affect their DNA-binding properties. As reported previously [17, 22], the introduction of rutinose on the 3-position of quercetin could cause steric hindrance, which may contribute to the unusual decrease in the DNA-binding affinity of rutin. In addition, a detailed comparison of the fractionbound values obtained for the isoflavone glycosides daidazin and puerarin further confirmed that the glycosylation sites can affect the DNA-binding affinity. Taken together, the sugar chains conjugated on the aglycones may experience different spatial orientations as a result of their conjugation sites and/or their identities, and cause different changes to the DNA-binding properties of flavonoid glycosides.

The skeleton of the aglycones also has an impact on the flavonoids binding to the DNA duplexes. For example, the flavanone naringenin shows a higher fractionbound value, followed by the flavone apigenin, and their isoflavone counterpart genistein shows a relatively lower fraction-bound value. The case was more remarkable in the pair liquiritigenin/daidzein: the flavanone liquiritigenin exhibits a much higher fraction-bound 
value than that of its isoflavone counterpart daidazein. It is obviously seen that the flavone and flavanone show enhanced binding toward the duplexes than the isoflavones. As reported previously, the conjugation of ring B on the $\mathrm{C} 3$ position introduces nonplanarity to the isoflavone skeleton, which causes steric hindrance and reduces the isoflavones binding to the DNA duplexes [23]. In contrast, the steric hindrance is greatly reduced in the flavone and flavanone with ring $\mathrm{B}$ conjugated on the $\mathrm{C} 2$ position. In addition, the double bond between C2 and C3 of the flavone skeleton allows extended conjugation among the three rings and forms an integrated $\pi$-conjugated plane that may facilitate its binding to DNA [17, 19, 46, 47]. In the case of flavanone, the single bond between C2 and C3 of the skeleton allows free rotation of ring $\mathrm{B}$ relative to the plane defined by ring $A$ and ring $C[47,48]$, which may result in more favorable conformations for its interaction with DNA.

Besides the $4^{\prime}-\mathrm{OH}$ group, the other hydroxyl substitutions on the flavonoid aglycone can also affect their DNA-binding properties. For example, the presence of the $3^{\prime}-\mathrm{OH}$ group proved to cause a remarkable increase in the fraction of bound DNA as easily seen in the pair apigenin/luteolin. For the pair luteolin/quercetin, the exchange of a hydrogen atom by a hydroxyl group at the 3-position also increased the fraction-bound value. Similar to the $4^{\prime}-\mathrm{OH}$ group, these additional hydroxyl substitutions may enhance the DNA-binding affinities of the flavonoids through hydrogen bonds with the DNA duplexes. In the case of isoflavone, the presence of the 5-OH group proved to correlate with increased DNAbinding affinities, as demonstrated in the pairs daidzein/ genistein and daidzin/genistin. This observation corresponds well with the previous results $[12,16,19]$, and it has been suggested that the presence of $5-\mathrm{OH}$ in genistein (or genistin) facilitates the formation of a strong intramolecular hydrogen bond with the 4-keto group and adds a six-membered ring moiety to the structure, which may further promote the interactions between the isoflavones and DNA [23]. Then, the relatively weak DNA-binding affinity of daidzein compared with those of the other aglycones with the $4^{\prime}-\mathrm{OH}$ group may be a result of the steric hindrance of the isoflavone skeleton (as discussed earlier) and the lack of the $5-\mathrm{OH}$ group.

Among the flavonoids studied, vitexin shows the greatest relative DNA-binding affinity. The notable structural features that may contribute to its extensive complexation with DNA include the presence of the important 4'-OH group, the planar flavone skeleton, and the conjugated glucose on $\mathrm{C} 8$ of ring $\mathrm{A}$, which may be spatially well oriented to provide the most hydrogen bonding and van der Waals interactions with the DNA structures. The flavone glycoside baicalin shows the lowest fraction of bound DNA, which may be largely attributed to the lack of the important 4'-OH group. In addition, charge repulsion should also be considered in this case. As the only flavonoid with a negatively charged sugar chain, baicalin may experience coulom- bic repulsion with the anionic backbone of the DNA in solution and the gas phase, and thus reduce its binding to the duplexes.

The sequence selectivities of these flavonoids were evaluated on the basis of the relative binding affinities of each flavonoid toward the five duplexes with different GC and AT content. For each flavonoid, the fraction values of bound DNA obtained for the five duplexes were approximately the same (see inset of Figure 2 for an example), suggesting that these flavonoids exhibit minimal sequence selectivity.

\section{Concentration Effects}

The effects of ligand concentrations on the DNA complexation were also examined. Duplex 1 was selected as one representative DNA duplex, given that minimal sequence selectivities were observed for all these flavonoids. The concentration-dependent binding studies were undertaken using a series of solutions containing a fixed DNA concentration of $20 \mu \mathrm{M}$ and a variable flavonoid concentration of $20,40,80$, or $120 \mu \mathrm{M}$. As the flavonoid/DNA molar ratios were increased from 1:1 to $6: 1$, the relative abundances of the flavonoid/DNA complexes increased and higher binding stoichiometries were observed (data not shown). For example, when the quercetin/duplex ratio was $1: 1$, only the $1: 1$ complex was observed with low abundance. As the quercetin/duplex ratio was increased, the abundance of the 1:1 complex increased and the 2:1 quercetin/DNA complex emerged. When the quercetin/duplex ratio was increased to $6: 1$, the 3:1 quercetin/DNA complex was observed. Similar results were obtained for the other flavonoids. Generally, the total number of bound drugs was found to be dependent on the overall drug/ DNA ratio. The concentration-dependent binding of these flavonoids is similar to the results obtained for ligands that have several equivalent binding sites like some well-known intercalators [31, 32]. This might indicate an intercalating or nonspecific binding of these flavonoids to the duplexes.

\section{ESI-MS/MS of the Flavonoid/DNA Complexes}

Although full-scan ESI-MS is useful to evaluate the binding stoichiometries, relative binding affinities, and sequence selectivities of the ligands toward the DNA duplexes, it can provide little insight into the binding interactions between the ligands and DNA. Previous studies have demonstrated that the binding modes could be deduced from the fragmentation patterns of the drug/DNA complexes via collision-induced dissociation [5, 29, 39-44]. Here, ESI-MS/MS experiments were undertaken to examine the fragmentation patterns as well as the stabilities of the flavonoid/DNA complexes. The fragmentation patterns of the 5-charged flavonoid/DNA complexes were systematically investigated because these fragmentation patterns have proven more reflective of the drug/DNA interactions 
than those obtained for complexes with higher charge states in a quadrupole ion trap mass spectrometer [39].

For the 5-charged complexes containing flavonoid aglycones, the predominant fragmentation pathway was ejection of a neutral ligand, whereas the loss of a nucleobase was insignificant or not observed. An example of this characteristic fragmentation pattern is shown in Figure 3a for the 1:1 complexes containing duplex 1 and quercetin. This fragmentation pattern is the same as that obtained for the well-known anthrapyrazole intercalators [43], suggesting that these flavonoid aglycones are likely to bind to the DNA duplexes via intercalation. Moreover, the low abundances of product ions resulted from base loss are indicative of weaker DNA-binding affinities of the flavonoid aglycones than those conventional intercalators previously studied [5, 29, 39]. The intercalative binding mode and weak binding affinities of the flavonoid aglycones toward DNA deduced from the tandem mass spectrometry are in fairly good agreements with the results obtained using solution-phase techniques [20, 22, 24].

The fragmentation patterns of the 5-charged complexes containing flavonoid glycosides are more complicated than those for the flavonoid aglycones. Generally, the losses of a neutral ligand and nucleobase occurred simultaneously. However, the extents of these fragmentation pathways depend on the flavonoid glycoside studied. For the flavone glycoside vitexin, the flavonol glycosides quercetrin, hyperin, rutin, and the flavanone glycoside naringin, the complexes underwent the predominant loss of nucleobase, whereas ejection of a neutral ligand occurred to a lesser extent (Figure $3 b$ and $d$ ). In the case of the flavanone glycoside liquiritin and the isoflavone glycosides puerarin, daidzin, and genistin, loss of a neutral ligand was the major fragmentation pathway, whereas base loss occurred to a lesser extent (Figure 3c). Unfortunately, a tandem mass spectrum of the complex with the flavone glucuronide baicalin was not obtained because of insufficient ion abundances. It should be noted that, for complexes containing the flavonol diglycoside rutin, loss of an anion ligand was also observed via collision-induced dissociation (Figure 3d); presumably, the 4-charged duplex is also formed; however, the $m / z$ of this ion lies out of the detectable mass range of the instrument.

The increased losses of nucleobase from the complexes with flavonoid glycosides in the tandem mass spectra suggest a different DNA-binding mode or stronger DNA-binding interactions of these flavonoid glycosides compared to that of their aglycone counterparts. Then, the relative DNA-binding affinities of the flavonoids were taken into account. For the flavone, flavanone, and isoflavone glycosides, the increased nucleobase losses seem to correlate well with their increased DNA-binding affinity. In this context, stronger binding interactions of these flavonoid glycosides toward the duplexes than their aglycone counterparts are more likely. However, for complexes containing the flavonol glycosides, the fragmentation patterns were quite different from that obtained for their aglycone counterpart quercetin, whereas the relative DNAbinding affinities underwent less change. In this case, the increased base losses in the tandem mass spectra are more likely to be a result of distinct DNA-binding modes of these flavonol glycosides from that of their aglycone counterparts.

\section{Effect of Glycosylation on the Flavonoids Binding to DNA Duplexes}

To further explore the effect of glycosylation on the flavonoids binding to DNA duplexes, the mixture containing a $4: 1 \mathrm{M}$ ratio ( 80 and $20 \mu \mathrm{M}$, respectively) of glucose and duplex 1 was prepared and examined using negative-ion ESI-MS. No complex ions were observed in the mass spectrum (data not shown). Moreover, mass spectra obtained from solutions containing a 1:4:4 M ratio of DNA duplex, flavonoid aglycone, and glucose were almost identical to those obtained without glucose. These results suggested that the binding of glucose to the duplex was rather weak, which was inconsistent with the previous results [49]. Therefore, it is reasonable to assume that the enhanced binding of flavone, flavanone, and isoflavone glycosides over their aglycone counterparts did not result from simple addi-
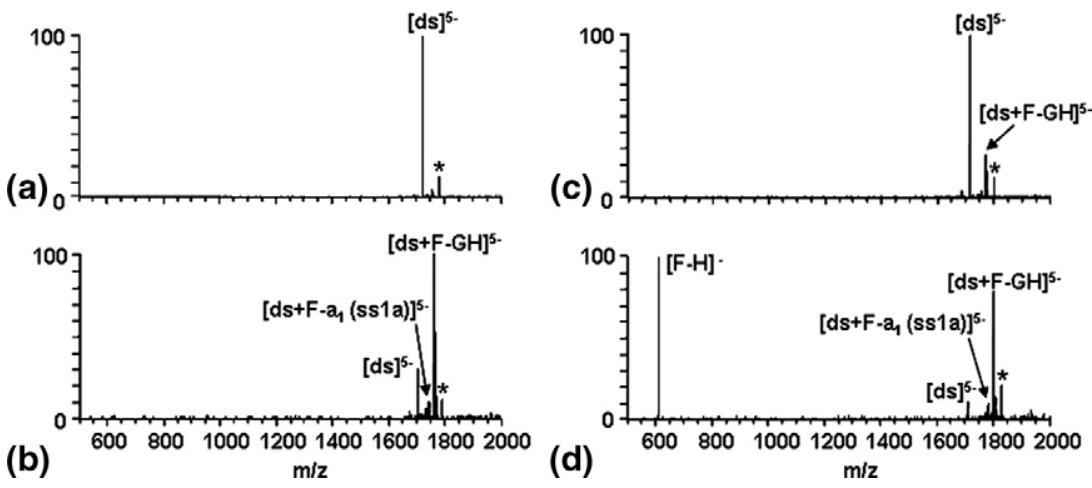

Figure 3. Negative ion ESI-MS/MS spectra of the 5-charged 1:1 complexes of duplex 1 with (a) quercetin; (b) vitexin; (c) genistin; and (d) rutin. Precursor ions are labeled with an asterisk. 
tion of the binding affinity of each part of the molecules. The entity of the molecules with sugar chain covalently linked onto the aglycones was necessary for the enhanced binding toward the DNA duplexes. Although the exact binding site and the mode of interaction between these flavonoid glycosides and DNA have not yet been resolved, we assume that the sugar parts of these flavone, flavanone, and isoflavone glycosides are attached to some parts of the DNA molecule and lead to enhanced binding affinities. Based on the previous studies of the interactions between sugar-containing drugs and DNA [50-53], the sugar chain in these flavonoid glycosides may interact with the minor grooves or form hydrogen bonds through the sugar $\mathrm{OH}$ groups with the backbone phosphate units, and then cause enhanced binding toward the DNA duplexes.

In the case of flavonols, glycosylation on the $3-\mathrm{OH}$ group did not result in significant changes of the DNA-binding affinities, indicating that the additional sugar chains engaged in DNA binding to a lesser extent or the binding mode might be altered because of the addition of sugar chain. Since the fragmentation patterns of complexes with these flavonol glycosides were quite different from those obtained for their aglycone counterpart, it is more favorable that the sugar chains conjugated on the 3-OH group result in a large alteration of the DNA-binding mode.

\section{Conclusion}

This study demonstrates ESI-MS is a powerful tool to evaluate noncovalent interactions between flavonoids and DNA duplexes. Binding stoichiometries, relative binding affinities, and sequence selectivities of the flavonoids toward the DNA duplexes can be rapidly estimated using negative-ion ESI-MS, and tandem mass spectrometry may provide useful information on the ligand-binding interactions. Our results have indicated that the $4^{\prime}-\mathrm{OH}$ groups of flavonoid aglycones were of primary importance for their DNA-binding properties and that flavonoid glycosides with sugar chain linked on ring $\mathrm{A}$ or ring $\mathrm{B}$ exhibited enhanced binding toward the duplexes over their aglycone counterparts. The aglycone skeleton as well as the other hydroxyl groups on the aglycone also exert an impact on the flavonoids binding to the duplexes. These results are expected to provide a deeper insight into the DNA-binding properties of flavonoids and useful guidelines for design of efficient anticancer agents.

\section{Acknowledgments}

The authors gratefully acknowledge the financial support from the National Natural Science Foundation of China (Grants 30672600 and 30472134) and the Great Research Project of Chinese Academy of Sciences (KGCX2-SW-213-06). The authors thank Associate Prof. Xinhua Guo and Ms. Cuihong Wan for their help and useful discussion during the work.

\section{References}

1. Tse, W. C.; Boger, D. L. Sequence-Selective DNA Recognition: Natural Products and Nature's Lessons. Chem. Biol. 2004, 11, 1607-1617.

2. Gniazdowski, M.; Denny, W. A.; Nelson, S. M.; Czyz, M. Effects of Anticancer Drugs on Transcription Factor-DNA Interactions. Expert Opin. Ther. Targets 2005, 9, 471-489.

3. Chen, W. H.; Qin, Y.; Cai, Z. W.; Chan, C. L.; Luo, G. A.; Jiang, Z. H. Spectrometric Studies of Cytotoxic Protoberberine Alkaloids Binding to Double-Stranded DNA. Bioorg. Med. Chem. 2005, 13, 1859-1866.

4. Song, F. R.; Liu, N.; Yan, C. Y.; Liu, Z. Q.; Liu, S. Y. Non-Covalent Interaction Between Oligonucleotide and Alkaloid Compounds via Negative Ion Electrospray Mass Spectrometry (I). Chem. J. Chin. Univ. 2004, 25, 1830-1832.

5. Wang, Z. F.; Guo, X. H.; Liu, Z. Q.; Cui, M.; Song, F. R.; Liu, S. Y. Studies on Alkaloids Binding to GC-Rich Human Survivin Promoter DNA Using Positive and Negative Ion Electrospray Ionization Mass Spectrometry. J. Mass Spectrom. 2008, 43, 327-335.

6. Qin, Y.; Pang, J. Y.; Chen, W. H.; Zhao, Z. Z.; Liu, L.; Jiang, Z. H. Inhibition of DNA Topoisomerase I by Natural and Synthetic Monoand Dimeric Protoberberine Alkaloids. Chem. Biodivers. 2007, 4, 481-487.

7. Gornall, K. C.; Samosorn, S.; Talib, J.; Bremner, J. B.; Beck, J. L. Selectivity of an Indolyl Berberine Derivative for Tetrameric G-Quadruplex DNA. Rapid Commun. Mass Spectrom. 2007, 21, 1759-1766.

8. Havsteen, B. H. The Biochemistry and Medical Significance of the Flavonoids. Pharmacol. Therapeut. 2002, 96, 67-202.

9. Iwashina, T. The Structure and Distribution of the Flavonoids in Plants. J. Plant Res. 2000, 113, 287-299.

10. Ren, W. Y.; Qiao, Z. H.; Wang, H. W.; Zhu, L.; Zhang, L. Flavonoids Promising Anticancer Agents. Med. Res. Rev. 2003, 23, 519-534.

11. Austin, C. A.; Patel, S.; Ono, K.; Nakane, H.; Fisher, L. M. Site-specific DNA Cleavage by Mammalian DNA Topoisomerase II Induced by Novel Flavone and Catechin Derivatives. Biochem. J. 1992, 282, 883-889.

12. Constantinou, A.; Mehta, R.; Runyan, C.; Rao, K.; Vaughan, A.; Moon, R. Flavonoids as DNA Topoisomerase Antagonists and Poisons: StructureActivity Relationships. J. Nat. Prod. 1995, 58, 217-225.

13. Boege, F.; Straub, T.; Kehr, A.; Boesenberg, C.; Christiansen, K.; Andersen, A.; Jakob, F.; Kohrle, J. Selected Novel Flavones Inhibit the DNA Binding or the DNA Religation Step of Eukaryotic Topoisomerase I. J. Biol. Chem. 1996, 271, 2262-2270.

14. Bernard, F. X.; Sable, S.; Cameron, B.; Provost, J.; Desnottes, J. F.; Crouzet, J.; Blanche, F. Glycosylated Flavones as Selective Inhibitors of Topoisomerase IV. Antimicrob. Agents Chemother. 1997, 41, 992-998.

15. Lopez-Lazaro, M.; Martin-Cordero, C.; Toro, M. V.; Ayuso, M. J. Flavonoids as DNA Topoisomerase I Poisons. J. Enzyme Inhib. Med. Chem. 2002, 17, 25-29.

16. Snyder, R. D.; Gillies, P. J. Evaluation of the Clastogenic, DNA Intercalative, and Topoisomerase II-Interactive Properties of Bioflavonoids in Chinese Hamster V79 Cells. Environ. Mol. Mutagen. 2002, 40, 266-276.

17. Webb, M. R.; Ebeler, S. E. Comparative Analysis of Topoisomerase I $\beta$ Inhibition and DNA Intercalation by Flavonoids and Similar Compounds: Structural Determinates of Activity. Biochem. J. 2004, 384, 527-541.

18. Das, B. B.; Sen, N.; Roy, A.; Dasgupta, S. B.; Ganguly, A.; Mohanta, B. C.; Dinda, B.; Majumder, H. K. Differential Induction of Leishmania donovani Bi-Subunit Topoisomerase I-DNA Cleavage Complex by Selected Flavones and Camptothecin: Activity of Flavones against Camptothecin-Resistant Topoisomerase I. Nucleic Acids Res. 2006, 34, 1121-1132.

19. Bandele, O. J.; Osheroff, N. Bioflavonoids as Poisons of Human Topoisomerase II $\alpha$ and II $\beta$. Biochemistry 2007, 46, 6097-6108.

20. Kanakis, C. D.; Tarantilis, P. A.; Polissiou, M. G.; Diamantoglou, S. Tajmir-Riahi, H. A. An Overview of DNA and RNA Bindings to Antioxidant Flavonoids. Cell Biochem. Biophys. 2007, 49, 29-36.

21. Bi, S. Y.; Qiao, C. Y.; Song, D. Q.; Tian, Y.; Gao, D. J.; Sun, Y.; Zhang, H. Q. Study of Interactions of Flavonoids with DNA Using Acridine Orange as a Fluorescence Probe. Sensor Actuat. B Chem. 2006, 119, 199-208.

22. Solimani, R. The Flavonols Quercetin, Rutin and Morin in DNA Solution: UV-Vis Dichroic (and Mid-Infrared) Analysis Explain the Possible Association between the Biopolymer and a Nucleophilic Vegetable-Dye. Biochim. Biophys. Acta 1997, 1336, 281-294.

23. Bocian, W.; Kawecki, R.; Bednarek, E.; Sitkowski, J.; Ulkowska, A. Kozerski, L. Interaction of Flavonoid Topoisomerase I and II Inhibitors with DNA Oligomers. New J. Chem. 2006, 30, 467-472.

24. Zhu, Z. W.; Li, C.; Li, N. Q. Electrochemical Studies of Quercetin Interacting with DNA. Microchem. J. 2002, 71, 57-63.

25. Kuzuhara, T.; Sei, Y.; Yamaguchi, K.; Suganuma, M.; Fujiki, H. DNA and RNA as New Binding Targets of Green Tea Catechins. J. Biol. Chem. 2006, 281, 17446-17456.

26. Beck, J. L.; Colgrave, M. L.; Ralph, S. F.; Sheil, M. M. Electrospray Ionization Mass Spectrometry of Oligonucleotide Complexes with Drugs, Metals, and Proteins. Mass Spectrom. Rev. 2001, 20, 61-87.

27. Hofstadler, S. A.; Sannes-Lowery, K. A. Applications of ESI-MS in Drug Discovery: Interrogation of Noncovalent Complexes. Nat. Rev. Drug Discov. 2006, 5, 585-595.

28. Wan, K. X.; Shibue, T.; Gross, M. L. Noncovalent Complexes between DNA-Binding Drugs and Double Stranded Oligodeoxynucleotides: A 
Study by ESI Ion Trap Mass Spectrometry. J. Am. Chem. Soc. 2000, 122, 300-307.

29. Wan, K. X.; Gross, M. L.; Shibue, T. Gas-phase Stability of DoubleStranded Oligodeoxynucleotides and Their Noncovalent Complexes with DNA-Binding Drugs Revealed by Collisional Activation in an Ion Trap. J. Am. Soc. Mass Spectrom. 2000, 11, 450-457.

30. Gale, D. C. Smith, R. D. Characterization of Noncovalent Complexes Formed Between Minor Groove Binding Molecules and Duplex DNA by Electrospray Ionization Mass Spectrometry. J. Am. Soc. Mass Spectrom. 1995, 6, 1154-1164.

31. Kapur, A.; Beck, J. L.; Sheil, M. M. Observation of Daunomycin and Nogalamycin Complexes with Duplex DNA Using Electrospray Ionization Mass Spectrometry. Rapid Commun. Mass Spectrom. 1999, 13, 24892497.

32. Gabelica, V.; De Pauw, E.; Rosu, F. Interaction between Antitumor Drugs and a Double Stranded Oligonucleotide Studied by Electrospray Ionization Mass Spectrometry. J. Mass Spectrom. 1999, 34, 1328-1337.

33. Gupta, R.; Kapur, A.; Beck, J. L.; Sheil, M. M. Positive Ion Electropray Ionization Mass Spectrometry of Double-Stranded DNA/Drug Complexes. Rapid Commun. Mass Spectrom. 2001, 15, 2472-2480.

34. Rosu, F.; Gabelica, V.; Houssier, C.; De Pauw, E. Determination of Affinity, Stoichiometry, and Sequence Selectivity of Minor Groove Complexes with Double Stranded Oligodeoxynucleotides by Electrospray Ionization Mass Spectrometry. Nucleic Acids Res. 2002, 30, e82.

35. Guittat, L.; Alberti, P.; Rosu, F.; Van Miert, S.; Thetiot, E.; Pieters, L.; Gabelica, V.; De Pauw, E.; Ottaviani, A.; Riou, J. F.; Mergny, J. L. Interactions of Cryptolepine and Neocrptolepine with Unusual DNA Structures. Biochimie 2003, 85, 535-547.

36. Oehlers, L.; Mazzitelli, C.; Rodriguez, M.; Brodbelt, J. S.; Kerwin, S. Evaluation of Complexes of DNA Duplexes and Novel Benzoxazoles or Benzimidazoles by Electrospray Ionization Mass Spectrometry. J. Am. Soc. Mass Spectrom. 2004, 15, 1593-1603.

37. Zhou, J.; Yuan, G. Analysis of Noncovalent Complexes between Human Telomeric DNA and Polyamides Containing N-Methylpyrrole and N-Methylimidazole by Using Electrospray Ionization Mass Spectrometry. Chem. Eur. J. 2005, 11, 1157-1162.

38. Ramos, C. I. V.; Barros, C. M.; Fernandes, A. M. Santana-Marques, M. G.; Correia, A. J. F.; Tome, J. P. C.; Carrilho, M. D. T.; Faustino, M. A. F.; Tome, A. C.; Neves, M. G. P. M. S.; Cavaleiro, J. A. S. Interactions of Cationic Porphyrins with Double-Stranded Oligodeoxynucleotides: A Study by Electrospray Ionisation Mass Spectrometry. J. Mass Spectrom. 2005, 40, 1439-1447.

39. Keller, K. M.; Zhang, J. M.; Oehlers, L.; Brodbelt, J. S. Influence of Initial Charge State on Fragmentation Patterns of Non-Covalent Drug/DNA Duplex Complexes. J. Mass Spectrom. 2005, 49, 1362-1371.

40. Rosu, F.; Pirotte, S.; De Pauw, E.; Gabelica, V. Positive and Negative Ion Mode ESI-MS and MS/MS for Studying Drug-DNA Complexes. Int. J. Mass Spectrom. 2006, 253, 156-171.
41. Mazzitelli, C. L.; Brodbelt, J. S.; Kern, J. T.; Rodriguez, M.; Kerwin, S. M. Evaluation of Binding of Perylene Diimide and Benzannulated Perylene Diimide Ligands to DNA by Electrospray Ionization Mass Spectrometry. J. Am. Soc. Mass Spectrom. 2006, 17, 593-604.

42. Mazzitelli, C. L.; Chu, Y. J.; Reczek, J. J.; Iverson, B. L.; Brodbelt, J. S. Screening of Threading Bis-Intercalators Binding to Duplex DNA by Electrospray Ionization Tandem Mass Spectrometry. J. Am. Soc. Mass Spectrom. 2007, 18, 311-321.

43. Smith, S. I.; Guziec, L. J.; Guziec, F. S., Jr.; Hasinoff, B. B.; Brodbelt, J. S Evaluation of Relative DNA Binding Affinities of Anthrapyrazoles by Electrospray Ionization Mass Spectrometry. J. Mass Spectrom. 2007, 42, 681-688.

44. Rosu, F.; Pirotte, S.; De Pauw, E.; Gabelica, V. Ligand Binding Mode to Duplex and Triplex DNA Assessed by Combining Electrospray Tandem Mass Spectrometry and Molecular Modeling. J. Am. Soc. Mass Spectrom. 2007, 18, 1052-1062.

45. Li, W.; Zhang, M.; Zhang, J. L.; Li, H. Q.; Zhang, X. C.; Sun, Q.; Qiu, C. M. Interactions of Daidzin with Intramolecular G-Quadruplex. FEBS Lett. 2006, 580, 4905-4910.

46. Yan, C. Y.; Liu, S. Y.; Zhou, Y. H.; Song, F. R.; Cui, M.; Liu, Z. Q. A Study of Isomeric Diglycosyl Flavonoids by SORI CID of Fourier Transform Ion Cyclotron Mass Spectrometry in Negative Ion Mode. J. Am. Soc. Mass Spectrom. 2007, 18, 2127-2136.

47. Zhang, J. M.; Brodbelt, J. S. Gas-Phase Hydrogen/Deuterium Exchange and Conformations of Deprotonated Flavonoids and Gas-Phase Acidities of Flavonoids. J. Am. Chem. Soc. 2004, 126, 5906-5919.

48. Zhang, J. M.; Brodbelt, J. S.; Wang, J. M. Threshold Dissociation and Molecular Modeling of Transition Metal Complexes of Flavonoids. J. Am. Soc. Mass Spectrom. 2005, 16, 139-151.

49. El-Mabdaoui, L.; Neault, J. F.; Tajmir-Riabi, H. A. CarbohydrateNucleotide Interaction. The Effects of Mono- and Disaccharides on the Solution Structure of AMP, dAMP, ATP, GMP, dGMP, and GTP Studied by FTIR Difference Spectroscopy. J. Inorg. Biochem. 1997, 65 123-131.

50. Hawley, R. C.; Kiessling, L. L.; Schreider, S. L. Model of the Interactions of Calichemicin $\gamma_{1}$ with a DNA Fragment from pBR322. Proc. Natl. Acad. Sci. U.S.A. 1989, 86, 1105-1109.

51. Uesugi, M.; Sekida, T.; Matsuki, S.; Sugiura, Y. Selective DNA Cleavage by Elsamicin A and Switch Function of Its Amino Sugar Group. Biochemistry 1991, 30, 6711-6715

52. Temperini, C.; Cirilli, M.; Aschi, M.; Ughetto, G. Role of the Amino Sugar in the DNA Binding of Disaccharide Anthracyclines: Crystal Structure of the Complex MAR70/d(CGATCG). Bioorg. Med. Chem. 2005, 13, 1673-1679.

53. Martin, J. N.; Munoz, E. M.; Schwergold, C.; Souard, F.; Asensio, J. L.; Jimenez-Barbero, J.; Canada, J.; Vicent, C. Carbohydrate-Based DNA Ligands: Sugar-Oligoamides as a Tool to Study Carbohydrate-Nucleic Acid Interactions. J. Am. Chem. Soc. 2005, 127, 9518-9533. 